\title{
25. DÉTERMINATION D'ORBITES PARABOLIQUES À PARTIR DE $N$ OBSERVATIONS AU MOYEN DE L'ORDINATEUR ÉLECTRONIQUE
}

\author{
H. DEBEHOGNE \\ Observatoire Royal de Belgique, Bruxelles, Belgique
}

\begin{abstract}
The Olbers-Banachiewicz method for computing parabolic orbits has been programmed. A least-square improvement of the orbit is obtained by varying the first and last geocentric distances.
\end{abstract}

\section{Introduction}

Dans le cas des astéroïdes et comètes, l'étude des écarts entre l'observation d'une part et d'autre part le calcul, dérivant des éléments d'une première orbite, permet de sélectionner parmi toutes les observations les trois qui serviront de base à l'établissement d'une orbite meilleure, sans perdre de vue pour autant que cette sélection reste soumise à l'examen qualitatif des clichés.

A l'Observatoire Royal de Belgique, c'est la méthode de Olbers-Banachiewicz (Stracke, 1929, p. 171) qui a été utilisée par Arend et ses collaborateurs.

\section{La première approximation}

L'étape initiale consiste à rechercher, au moyen de la règle de fausse position, la distance $\Delta_{1}$ de la comète à la Terre à l'instant correspondant à la première observation de base. On sait que cette méthode utilise au départ deux valeurs approchées de $\Delta_{1}$. Banachiewicz obtient ces deux valeurs à partir d'une valeur approchée de $\Delta_{1}$ à moins de 0,01 à laquelle il ajoute et retranche 0,01 . Les calculs doivent conduire à obtenir l'égalité entre le déplacement héliocentrique géométrique $S_{g}$ et le déplacement héliocentrique dynamique $S_{d}$ de la comète.

Ce déplacement dynamique est obtenu grâce à la formule tirée du théorème de Lambert (Stracke, 1929, p. 35). Banachiewicz trouve par exemple $S_{g}-S_{d}=+0,00001$ à partir de $\Delta_{1}=1,71252$ (comète 1925c). L'ordinateur effectue les calculs avec 16 chiffres significatifs et il donne pour $\Delta_{1}=1,71194$ une valeur $S_{g}-S_{d}$ vérifiant $S_{g}-S_{d}<$ 0,000001003 . Cet écart important entre les 2 valeurs de $\Delta_{1}$ a été déjà rencontré entre les 2 valeurs de la solution de l'équation de Képler en sinus hyperbolique. La première avait été obtenue en utilisant 8 chiffres significatifs (comme le permettent seulement les tables du sinus hyperbolique). La deuxième l'avait été en utilisant 16 chiffres significatifs comme dans notre programme de calcul des résidus pour une orbite hyperbolique (Debehogne, 1968). Comme, en fin de calcul, nos résidus relatifs à la seconde observation sont moitié moindres $(+0,3$ et $-0,8)$ que ceux de Banachiewicz on peut considérer que notre valeur de $\Delta_{1}$ est meilleure que celle de l'exemple exposé par Stracke. Il convient de noter que cet écart porte déjà sur le quatrième chiffre décimal alors que l'auteur travaille constamment avec cinq chiffres décimaux. 


\section{Valeur approchée de $\Delta_{1}$}

L'obtention de la première valeur approchée de $\Delta_{1}(1,71)$ exige la connaissance d'une valeur approchée de $\Delta_{2}$ (distance comète-Terre à l'instant correspondant à la deuxième observation de base). Cette valeur doit vérifier l'équation $(\mathrm{CR})^{2} \mathrm{CS}-c=0$, où $c$ est égal à $2 d^{2} R_{2}^{2}, R_{2}$ étant la distance Terre-Soleil à l'instant correspondant à la deuxième observation de base et $d$ valant $g(1+M) / 2 h, M$ étant fonction des instants des 3 observations de base, des coordonnées du Soleil à l'instant de la seconde observation et des coordonnées géocentriques de la comète, $g$ et $h$ étant liés respectivement au déplacement de la Terre et de la comète.

Quant à CR et CS on voit leur signification sur le graphique (Stracke, 1929, p. 183) qui servait à Banachiewicz pour déterminer $\Delta_{2}$ à moins de 0,01 .

Nous introduisons, par une carte contenant certaines données, la première valeur approchée de $\Delta_{2}$ d'où l'ordinateur tire les valeurs de CS, RC et donc de $(\mathrm{CR})^{2} \mathrm{CS}-c$. Nous augmentons successivement $\Delta_{2}$ de $0,001 \times n(n=1,2,3, \ldots)$. Quand le signe de $(\mathrm{CR})^{2} \mathrm{CS}-c$ change nous retournons à l'avant-dernière valeur de $\Delta_{2}$ que nous augmentons successivement de $0,001 \times n / 2$ et ainsi de suite jusqu'à la détermination de $\Delta_{2}$ avec 2 décimales.

\section{Détermination des éléments}

Pour ne pas dépasser la capacité de l'ordinateur nous avons découpé le programme en programmes partiels, afin d'obtenir l'angle voulu puisque l'ordinateur donne pour $x=\operatorname{arctg} y$ des valeurs comprises entre $-\pi / 2$ et $+\pi / 2$.

Nous obtenons ainsi sur cartes les éléments suivants: l'instant de passage $T$ au périhélie, le noeud $\Omega$, la distance $\omega$ du $\Omega$ au périhélie, l'inclinaison $i$ du plan de l'orbite sur l'écliptique et la distance périhélique $q$.

\section{Les résidus}

Le but poursuivi au cours de ce travail est le calcul des 108 résidus correspondant aux observations de la comète 1957 III (Arend-Roland), effectuées à Uccle.

On peut recourir à la méthode décrite plus haut à propos de la résolution de $(\mathrm{CR})^{2} \mathrm{CS}-c=0$ ou utiliser la méthode de fausse position comme ci-dessus pour le calcul de $\Delta_{1}$ ou encore programmer la formule de Lemaître suivant le procédé exposé par Arend (1966). Nous avons préféré la méthode suivante qui nous paraissait la plus rapide: soit à résoudre l'équation

$$
x+\frac{x^{3}}{3}=\frac{k}{\sqrt{2}} \frac{t-T}{q^{3 / 2}}
$$

ou $x=\operatorname{tg} v_{1} / 2, k=0,01720209895$, et $t=$ instant d'observation. Si on pose successivement

$$
\begin{aligned}
& x=2 \operatorname{cotg} 2 y \\
& y=\sqrt[3]{\operatorname{tg} \frac{z}{2}},
\end{aligned}
$$


l'équation s'écrit

$$
\operatorname{tg} z=\frac{2 \sqrt{2}}{3 k} \frac{1}{M}
$$

\section{Conclusion}

On calcule d'abord une orbite préliminaire à partir de trois observations de base et les résidus de toutes les observations pour lesquelles on dispose d'un résultat précis: on calcule d'abord la distance Terre-comète $\Delta$ à partir de $t-A, A$ étant la constante de lumière; $\Delta$ est donc supposé égal à l'unité, puis on corrige $t$ de $-A \Delta$, d'où en réalité deux calculs, alors qu'auparavant on se contentait d'une règle de trois à partir de $A \Delta_{1}, A \Delta_{2}, A \Delta_{3}$, où $\Delta_{1}, \Delta_{2}, \Delta_{3}$ sont les distances astre-Soleil pour les trois observations de base. Les résultats varient peu pour $\varepsilon=\varepsilon+\Delta \varepsilon$, avec $\Delta \varepsilon$ valant environ vingt secondes d'arc ( $\varepsilon=$ inclinaison de l'équateur sur l'écliptique).

Enfin, on améliore l'orbite par la méthode de variation des distances $\Delta_{1}$ et $\Delta_{3}$ et application des moindres carrés.

\section{Réferences}

Arend, S.: 1966, Commun. Astron. Obs. Roy. Belg. No. 243.

Debehogne, H.: 1968, Acad. Roy. Belg. Bull. Cl. Sci. 54, 941.

Stracke, G.: 1929, Bahnbestimmung der Planeten und Kometen, Springer, Berlin. 